\title{
Electrolyte Thin Film Formation for Solid Oxide Fuel Cells Using Water-based Slurry Contained $\mathrm{Ce}_{0.9} \mathrm{Gd}_{0.1} \mathrm{O}_{1.95}$ Nano-powder
}

\author{
Shin-ichi Hashimoto * and Masashi Mori
}

\author{
Materials Science Research Laboratory, Central Research Institute of Electric Power Industry (CRIEPI, 2-6-1 \\ Nagasaka, Yokosuka, Kanagawa 240-0196, Japan) \\ (* presence affiliation: Center for Research Strategy and Support, Tohoku University)
}

Received August 28, 2008 ; Accepted January 16, 2009

\begin{abstract}
We developed environmental-friendly co-sintering processing technology of electrolyte/anode layers in solid oxide fuel cells (SOFCs) using several different types of nano-powders and water-based slurry. The shrinkages of $\mathrm{Ce}_{0.9} \mathrm{Gd}_{0.1} \mathrm{O}_{1.95}(\mathrm{CGO})$ nano-powder for electrolyte and NiO-CGO nano-powder for anode substrate matched each other after the appropriate material selection and the optimization of pre-treatments. Next, the water-based CGO slurry was developed for electrolyte thin film formation. By effect of an emulsion type binder and lemon pectin, the water-based CGO slurry which has the both adhesive strength and stability was obtained. Finally, a dense and thin CGO film was formed on the NiO-CGO anode substrate by co-sintering process at $1400^{\circ} \mathrm{C}$, and the prepared film thickness was ca. $8 \mu \mathrm{m}$.
\end{abstract}

Key Words : SOFC, Cosintering, Water-Based Slurry, Ceria-Based Oxides

\section{Introduction}

Solid oxide fuel cells (SOFCs) are expected as high efficiency electric power generators. In recent years, SOFCs operated at $500-650^{\circ} \mathrm{C}$ are extensively researched since it can lead to the use of low-cost alloys in SOFCs with a fast start-up. Metal supported planar SOFCs were developed by Brandon et al., and it could show $200 \mathrm{mWcm}^{-2}$ or more of power density at $550^{\circ} \mathrm{C}$. ${ }^{1,2)}$ Suzuki et al. succeeded in the remarkable improvement of micro-tubular SOFC performance, i.e., over $0.3 \mathrm{Wcm}^{-2}$ at $550{ }^{\circ} \mathrm{C}$, by introducing thin ceria based electrolyte into the cell .3)

One of the important challenges for such SOFCs is development of fabrication technology for thin electrolyte film formation on a porous electrode substrate. Since electrical conductivities of the solid electrolytes are quite lower, compared with other SOFC components, dense and thin electrolyte formation become important for power generation efficiency. A co-sintering method between a thin electrolyte and an electrode substrate is one of the candidates for SOFC production technology. ${ }^{4-6)}$ Slurry which contains fine electrolyte powder is homogeneously coated on the green electrode substrate, and the coated substrate is sintered after being dried for the densification of the film. By optimization of the process, it is possible to prepare dense electrolyte thin film on the electrode substrate.

However, there are two important issues. One is development of the slurry for the coating, and another is matching of shrinkage between the electrolyte and electrode substrate material. Organic solvent based slurry is wildly introduced for the pilot cell fabrication because of its high uniformity and adhesive strength. However, from an environmental viewpoint, the water-based slurry is desired for mass production although the development of water-based slurry is more difficult than that of organ- ic solvent based slurry. In the use of water-based slurry for electrolyte film formation, water soluble binders cannot be applied to the substrate forming due to water penetration from the slurry to the substrate. Therefore, substrate forming without water soluble binders is required, and the shrinkage of the green substrate must match with that of electrolyte powder.

The use of the nano ceramic powder has potential to solve these issues. If the dispersion of the nano-powder is held in the slurry, the homogeneous sintering process is expected. And moreover, since the nano-powders have high surface area, it is also possible to control the shrinkage of the powders by heat treatment, ball milling and so on.

In this study, we developed environmental-friendly cosintering processing technology for $\mathrm{Ce}_{0.9} \mathrm{Gd}_{0.1} \mathrm{O}_{1.95}$ (CGO) electrolyte formation on a NiO-CGO anode substrate using water-based slurry. The CGO and NiO-CGO nanopowders were introduced as raw materials of the electrolyte and anode substrate, respectively. The waterbased slurry of CGO was developed, and the shrinkage of NiO-CGO substrate was adjusted to that of the CGO nano-powder using NiO-CGO nano-powder. The NiO-CGO substrate was prepared without any binders, and we finally prepared the dense CGO electrolyte thin film on NiO-CGO electrode.

\section{Experimental}

The sintering property and micro structure of CGO and NiO-CGO powders were studied first for material selection. The shrinkages of these powders were confirmed from room temperature to $1600{ }^{\circ} \mathrm{C}$. The candidate powders were pressed into cylinders by uniaxial pressing at $23 \mathrm{MPa}$, and re-pressed by isostatical pressing at $200 \mathrm{MPa}$. The diameters of cylinders were measured 
Table 1 Specifications of the selected powders for cell fabrication.

\begin{tabular}{|c|c|c|c|}
\hline \multirow[t]{2}{*}{ Selected powders } & \multirow{2}{*}{$\begin{array}{c}\text { For electrolyte } \\
\mathrm{Ce}_{0.9} \mathrm{Gd}_{0.1} \mathrm{O}_{1.95}\end{array}$} & \multicolumn{2}{|c|}{ For anode substrate } \\
\hline & & $\mathrm{Ce}_{0.9} \mathrm{Gd}_{0.1} \mathrm{O}_{1.95}$ & $\mathrm{NiO}$ \\
\hline \multirow[t]{3}{*}{ Synthesis method } & Nano particle & & Closed \\
\hline & growth method & Citrate method & (Sumitomo Metal Mining, \\
\hline & (Co-precipitation) & & Powder code : Q42) \\
\hline Surface area $\left(\mathrm{m}^{2} / \mathrm{g}\right)$ & 32 & 32 & 107 \\
\hline Average particle size ${ }^{*}(\mu \mathrm{m})$ & 0.4 & 0.17 & 6.7 \\
\hline
\end{tabular}

* The particle size was measured by laser diffraction particle size analyzers.

after the heat-treatments at each set temperature for 10 hours, and the shrinkage was calculated on the basis of green cylinder diameter. The shrinkages of the materials as a function of temperature were obtained in this manner.

Table 1 shows specifications for the ultimately selected powders. From several candidates, the CGO powder which was prepared by the nano-particle growth technique $^{7)}$ is introduced for electrolyte formation. And the mixture of CGO powder prepared by citrate method and $\mathrm{NiO}$ powder was used for the anode substrate. The $\mathrm{NiO}$ powder from Sumitomo Metal Mining Co., Ltd., which is named "Q42" was used for the mixture. The $\mathrm{NiO}$ and CGO powders are sufficiently mixed each other using ball milling in the volume ratio of $\mathrm{NiO}: \mathrm{CGO}=46: 54$. The particles of the CGO powder for the electrolyte and the mixture of NiO-CGO powder for the anode substrate were observed by FE-TEM (Field Emission Transmission Electron Microscope, JEOL, JEM-2100F).

As the second step, the water-based slurry of CGO was developed. After simplified matching test with the CGO nano-powder, an emulsion type binder, "DB-20" from Yuken Industry Co., Ltd., Japan was selected from over 20 candidates. The CGO nano-powder $5 \mathrm{~g}, \mathrm{H}_{2} \mathrm{O}$ (distilled water) $15 \mathrm{ml}$, binder “DB-20” $1.667 \mathrm{~g}$ (solid content), anti-foam agent $0.01 \mathrm{~g}$ and dispersant $0.025 \mathrm{~g}$ are mixed each other by ball milling for over 48 hours. The water-based CGO slurry was prepared by this means. As a contraction control agent, the doping effect of lemon pectin to the CGO slurry has been also studied. Lemon pectin $0.2 \mathrm{~g}$ was doped into the slurry, and mixed by ball milling.

As the final stage, the CGO thin film has formed on a $\mathrm{NiO}-\mathrm{CGO}$ substrate. The mixture of $\mathrm{NiO}$ and CGO nanopowders were pressed into cylinders by uniaxial pressing at $80 \mathrm{MPa}$, and sintered at $700{ }^{\circ} \mathrm{C}$ for 5 hours for the substrate preparation. Any kind of binders were not used for this process. Surface of the NiO-CGO substrates were coated with the prepared CGO slurry by a paintbrush. After drying for 24 hours, the samples are sintered at $1400{ }^{\circ} \mathrm{C}$ for 10 hours. The sample surface and cross section were observed by FE-SEM (Field Emission Scanning Electron Microscope, Hitachi, S4500SE/N).

\section{Results and Discussion}

Figure 1 shows the shrinkages of CGO and NiO-CGO nano-powders from room temperature to $1600{ }^{\circ} \mathrm{C}$. In our

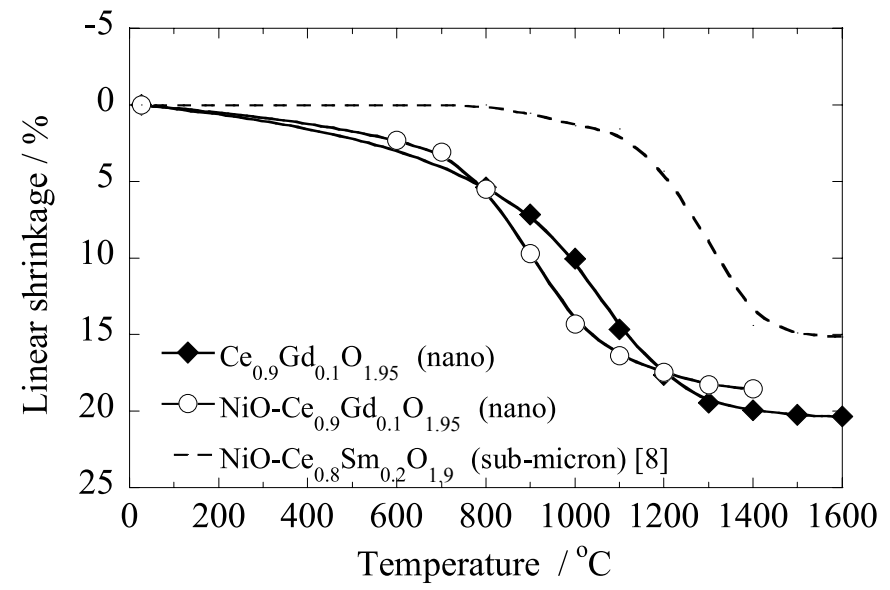

Fig. 1 Shrinkages of the selected CGO and NiO- CGO powders from room temperature to $1600{ }^{\circ} \mathrm{C}$.

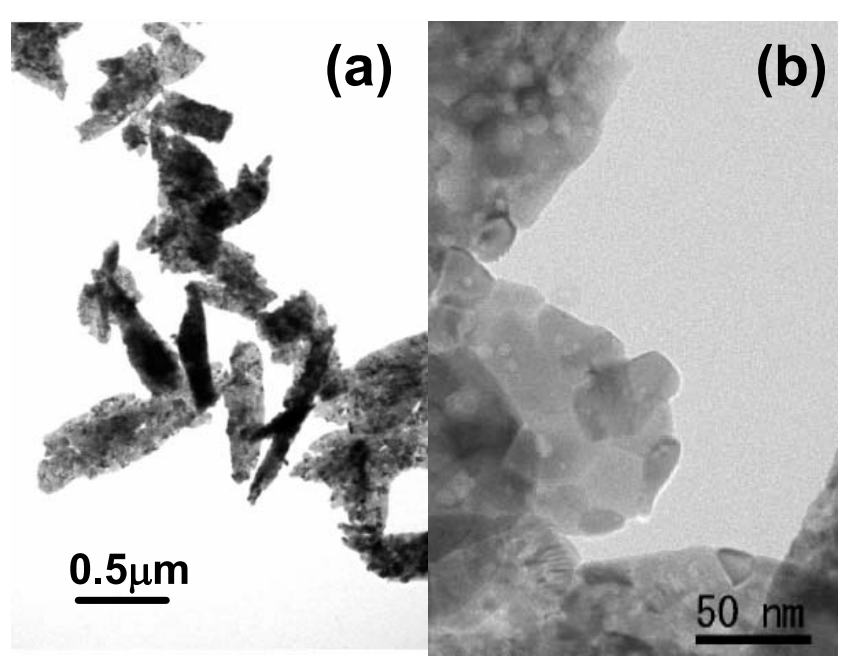

Fig. 2 TEM images of CGO powder for the electrolyte.

early research, we studied the mixture of $\mathrm{NiO}$ and $\mathrm{Ce}_{0.2} \mathrm{Sm}_{0.8} \mathrm{O}_{1.9}$ (CSO), which was processed by citrate method and had sub-micron particle size, for the substrate. ${ }^{8)}$ However, the densification of NiO-CSO powder only just started around $1200{ }^{\circ} \mathrm{C}$, and the shrinkage did not match with the CGO nanopowder. After the appropriate material selection and the optimization of pretreatments, the shrinkage of NiO-CGO powder was controlled as shown in Fig.1 and became almost comparable to that 
of CGO nano-powder for electrolyte formation.

Figure 2 shows the TEM images of CGO powder for the electrolyte, and Fig. 3 shows the TEM image of NiOCGO powder for the electrode substrate. The both powders had nano-scale primary particle size, approximately $20 \mathrm{~nm}$. These results were not accord to the average particle size in Table 1 . Since laser diffraction particle size analyzers were used for the particle size measurements, the particle sizes in Table 1 probably reflect their secondary particle sizes. Additionally, the mixture of $\mathrm{NiO}$ and CGO powder was treated by ball milling process. It means that the mixture has done not only mixing but also raveling. As the result, Fig. 3 was obtained. It is known that usual $\mathrm{NiO}$ and/or ceria which have submicron particle size require high sintering temperatures not less than $1500{ }^{\circ} \mathrm{C}$ as our initial study (dot line in Fig. 1). However, the densification of selected CGO and NiOCGO powders almost finished around $1200{ }^{\circ} \mathrm{C}$ and completed up to $1400{ }^{\circ} \mathrm{C}$. This lowering of the sintering temperature was caused by nano-sized micro structure. It will contribute to the fabrication flexibility for the SOFCs, the processing energy reduction and so on.

Using this NiO-CGO powder, the NiO-CGO substrate was prepared by just pressing and low temperature heat treatment $\left(700{ }^{\circ} \mathrm{C}\right.$ for 5 hours) without any binders. In spite of such low temperature heat-treatment, the prepared NiO-CGO substrate had enough strength for handling. The partial particle growths for $\mathrm{NiO}$ and CGO nano primary particles most likely contribute to the strength although we did not observe the microstructure.

Next, the water-based CGO slurry was developed for electrolyte thin film formation. One of the important key factors for water-based slurry is a binder. From viewpoint of the adhesive strength, we focused on emulsion type binders. To get homogeneous slurry, the selection of the suitable binder for the CGO nano-powder is required. After simplified matching test, an emulsion type binder, “DB-20" was selected from over 20 candidates. During the test, most of the emulsion type binders were not able to keep dispersion of the CGO powder in

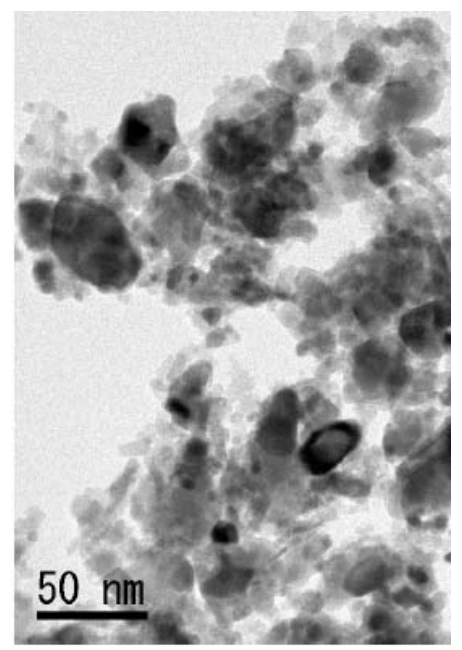

Fig. 3 TEM image of NiO- CGO powder for the electrode substrate. water. Since charge statuses near the surface of particles are easily changed by $\mathrm{pH}$ in water based slurry, ${ }^{9)}$ optimization of the slurry $\mathrm{pH}$ is important for high dispersion of the nano-powder. However, we found that "DB20" made this CGO nano-powder homogeneously dispersed without any $\mathrm{pH}$ control.

Then, water-based CGO slurry, which contains binder "DB-20", was prepared with mixing ratio as shown in "Experimental". After sufficient ball milling, the prepared slurry was coated on a NiO-CGO substrate for four times by a paint brush. Right after coating, the film on the NiO-CGO substrate seemed to be uniform. However, after 24 hours drying at room temperature, the film was peeling off from the edge (Fig. 4 (a)). Contraction of the film during the drying process seemed to cause this peeling problem at the edge. Therefore, control of the contraction was tried by adding other agent to this slurry. After trial and error, we found that lemon pectin worked as a contraction control agent. Figure 4 (b) shows CGO film which was coated by the lemon pectin doped CGO slurry. Even after 48 hours drying, the peeling of film was not observed, and the adhesive strength between the CGO film and NiO-CGO substrate was high enough for the handling.

Finally, the densification of the CGO film has done by co-sintering technique. The coated sample was sintered at $1400{ }^{\circ} \mathrm{C}$ for 10 hours. The shrinkages of CGO and $\mathrm{NiO}$ CGO powders matched each other as shown in Fig. 1, and the NiO-CGO substrate was prepared at relatively low temperature. Therefore, it is expected that the densified CGO film on the NiO-CGO substrate is obtained after the sintering process. Figure 5 shows the densified CGO film (a) the external appearance, (b) the magnified SEM image of the surface. As expected, the CGO film after co-sintering with the NiO-CGO substrate became transparent, i.e. the film was highly densified. The grain size of CGO has grown up to $c a .2 \mu \mathrm{m}$ on an average as shown in Fig. 5 (b). Figure 6 shows the cross section of the sample (a) SEM image and (b) REM (Reflection Electron Microscopy) image. In spite of coating by a paint brush, the uniformity of CGO thin-film was controlled well, and its average thickness was about $8 \mu \mathrm{m}$. Because just a few small closed pores were observed in the CGO thin-film, it will work as a gas-tight electrolyte.
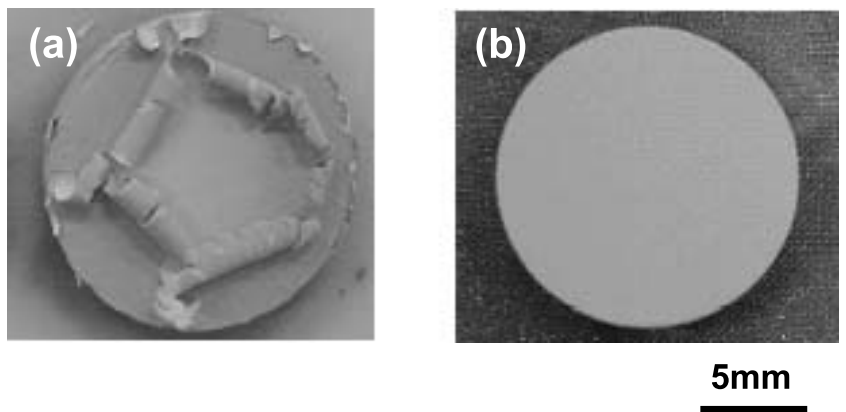

Fig. 4 CGO film coated by CGO slurry on NiO- CGO substrate. (After drying for 24 hours.)

(a) without lemon pectin, (b) doped lemon pectin. 

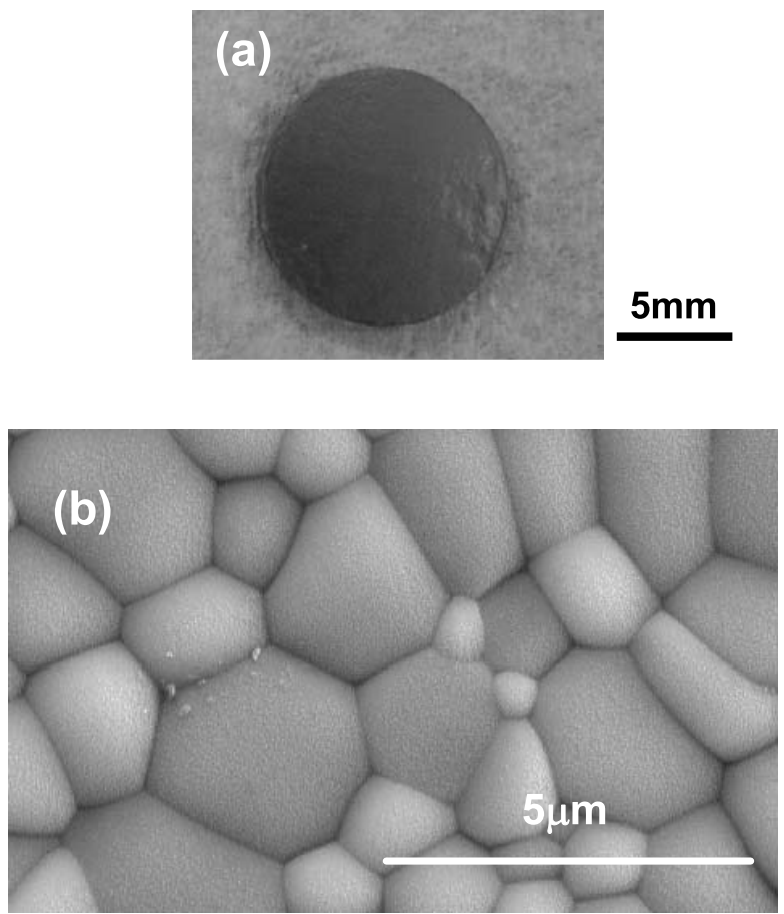

Fig. 5 Surface of the densified CGO film (a) external appearance, (b) magnified SEM image.
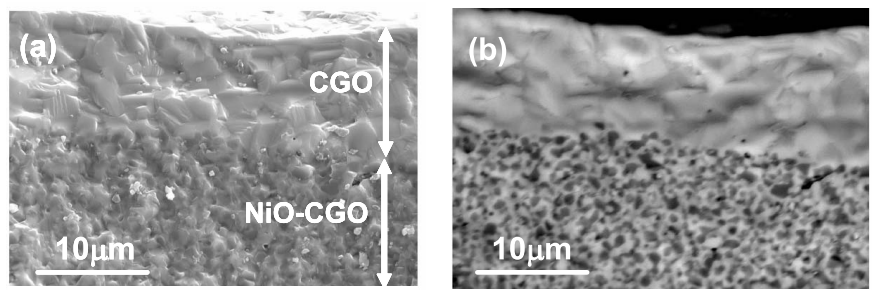

Fig. 6 Cross section of the sample (a) SEM image and (b) REM image.

The black dots in REM image (Fig. 6 (b)) suggest the intensity for $\mathrm{NiO}$. It is understood that $\mathrm{NiO}$ and $\mathrm{CGO}$ are well mixed each other in the NiO-CGO substrate. Such a substrate can be expected as a high performance anode since a large amount of three-phase boundary is produced after reduction of $\mathrm{NiO}$. Thus, by attachment of a cathode, e.g. $\mathrm{La}_{0.6} \mathrm{Sr}_{0.4} \mathrm{Fe}_{0.8} \mathrm{Co}_{0.2} \mathrm{O}_{3-\delta}$, this sample will work as a high performance fuel cell in the $450-600{ }^{\circ} \mathrm{C}$ temperature range.

\section{Conclusions}

We studied the CGO electrolyte film formation on the $\mathrm{NiO}$ - CGO anode substrate. Combination of several different nano-powders gave the good shrinkage matching between electrolyte and anode substrate materials at first. The water-based CGO slurry was developed for electrolyte thin film formation. By effect of an emulsion type binder and lemon pectin, the water-based CGO slurry which has the both adhesive strength and stability was obtained. Finally, we succeeded in densification of thin CGO film on NiO-CGO anode substrate using co-sintering process.

\section{Acknowledgments}

FE-TEM observation has done by Electric Power Engineering Systems Co., Ltd. This work had been supported by NEDO, Japan, as part of the Advanced Ceramic Reactor Project.

\section{References}

1) J. Larminie and A. Dicks, Fuel Cell System Explained $2^{\text {nd }}$ eds., John Wiley and Sons, Chichester, England, p.286(2003).

2) N. P. Brandon, A. Blake, D. Corcoran, D. Cumming, A. Duckett, K. El-Koury, D. Haigh, C. Kidd, R. Leah, G. Lewis, C. Matthews, N. Maynard, N. Oishi, T. McColm, R. Trezona, A. Selcuk, M. Schmidt, and L. Verdugo, J. Fuel Cell Science and Technology, 1-1, 61 (2004) .

3) T. Suzuki, T. Yamaguchi, Y. Fujishiro, and M. Awano, J. Electrochem. Soc., 153, A925 (2006).

4) I. Yasuda, Y. Baba, K. Ogasawara, H. Yakabe, and Y. Matsuzaki, in SOFC VII, H. Yokokawa, S. C. Singhal, Editors, PV2001-16, p.131, The Electrochemical Society, Proceedings Series, Pennington, 2001.

5) N. Christiansen, S. Kristensen, H. Holm-Larsen, P. H. Larsen, M. Mogensen, P. V. Hendriksen, and S. Linderoth, in SOFC VIII, S.C. Singhal, M. Dokiya Editors, PV200307, p.105, The Electrochemical Society, Proceedings Series, Pennington, 2003.

6) Y. Funahashi, T. Shimamori, T. Suzuki, Y. Fujishiro, and M. Awano, J. Power Sources, 163, 731 (2007).

7) E. Suda, M. Mori, K. Murai, B. Pacaud, and T. Moriga, J. the Ceramic Soc. Japan, 113, 793 (2005).

8) S. Hashimoto, M. Mori, E. Suda, T. Kunimoto, and T. Moriga, 19th Fall Meeting of the Ceramic Society of Japan, 2B19, Yamanashi University, Yamanashi, Japan, September, 2006.

9) C. Takai, M. Fuji and M. Takahashi, Annual report of the ceramics research laboratory Nagoya Institute of Technology, 6, 32 (2006). 